\title{
Monocyte-derived macrophages for treatment of cerebral palsy: a study of 57 cases
}

This article was published in the following Dove Press journal: Journal of Neurorestoratology

\author{
Elena Chernykh' \\ Ekaterina Shevela' \\ Marina Kafanova ${ }^{2}$ \\ Lyudmila Sakhno' \\ Evgeny Polovnikov ${ }^{2}$ \\ Alexandr Ostanin' \\ 'Laboratory of Cellular \\ Immunotherapy, Research Institute of \\ Fundamental and Clinical Immunology, \\ Novosibirsk, Russia, ${ }^{2}$ Department of \\ Neurosurgery, Children's City Clinical \\ Hospital No I, Novosibirsk, Russia
}

Correspondence: Ekaterina Shevela Research Institute of Fundamental and Clinical Immunology, Yadrintsevskaya Street, 14, Novosibirsk 630099, Russia

$\mathrm{Tel}+73832360329$

Emailshevelak@mail.ru
Objective: Cell-based technologies are considered to be a new approach for the treatment of cerebral palsy (CP). Given the potent anti-inflammatory activity and high regenerative potential of M2 macrophages, these cells may be a promising source for cell transplantation. To evaluate the safety and efficacy of autologous M2 macrophages, we performed an open-label, Phase I/ II, non-controlled clinical trial in children with severe CP.

Patients and methods: Fifty-seven children with severe CP, including 33 boys and 24 girls, with a median age of 4 years were enrolled in the study. The patients were treated with intrathecal administration of autologous M2 macrophages. The primary outcome measure was safety, and the secondary outcome measure was functional improvement in neurologic scales, including the 66-item Gross Motor Function Measure test, Peabody Developmental Motor Scale-Fine Motor test, Ashworth scale, Medical Resarch Council scale, and an easy-to-understand unified questionnaire for evaluation of cognitive functions in our modification.

Results: Intrathecal introduction of M2 cells in a median dose of $11.2 \times 10^{6}$ did not induce any serious adverse events either related with cell injection or during 5 years of follow-up. After 3 months, the Gross Motor Function Measure score increased from 19 \pm 4.5 to $77 \pm 7.8$, the Peabody Developmental Motor Scale-Fine Motor test score improved from $0.9 \pm 0.23$ to $4.4 \pm 0.51$, and the Ashworth score decreased from $3.5 \pm 0.11$ to $2.5 \pm 0.16$. The assessment of cognitive functions revealed an increase from $1.22 \pm 0.24$ to $3.98 \pm 0.95$, and a reduction of seizure syndrome was registered. In addition, M2 injection was accompanied by an increased production of brainderived neurotrophic factor $\left(\mathrm{p}_{\mathrm{U}}=0.015\right)$.

Conclusion: The data obtained suggest that cell therapy based on M2 macrophages is safe, does not induce any severe cell-related reactions or long-term side effects and comorbidities, and is accompanied by significant neurologic improvements in severe $\mathrm{CP}$ patients.

Keywords: cerebral palsy, clinical study, cell-based therapy, M2 macrophages, neurologic improvement

\section{Introduction}

Cerebral palsy (CP) is a non-progressive brain disorder, which is defined as a chronic motor disability with various etiologies. ${ }^{1}$ It is closely associated with development abnormalities or damage resulting from brain injury in utero or early infancy and is accompanied by disturbances of sensation, perception, cognition, communication, behavior, epilepsy, and secondary musculoskeletal problems. ${ }^{2-4}$ The pathophysiology of CP is still largely unclear due to its heterogeneity and lack of relevant animal models. The most often prevalent pathological lesion in CP connected with the damage of the corticospinal tracts is periventricular white matter injury and intraventricular 
hemorrhage. In both cases, inflammation is considered the leading pathogenic factor that induces neural cell damage. ${ }^{5-7}$ Convincing evidence has been obtained pointing to the association between inflammation and neonatal encephalopathy. ${ }^{8-10}$ Moreover, striking improvement in motor function following reduction of neuroinflammation in a rabbit model of CP demonstrates the key role of inflammation in the pathophysiology of CP. ${ }^{11}$

Currently, there are no effective treatments for $\mathrm{CP}$ which can substantially improve motor and cognitive functions, particularly in patients with moderate-to-severe disease, and the best available therapies aim at functional, social, and nutritional support. ${ }^{9,12}$

Given various disturbances in the brain tissue that involve several cell types and multiple mechanisms, CP seems to be an optimal target for cell therapy to repair the neurologic functions. ${ }^{1,13,14}$ Actually, motor improvements following stem cell administration were shown in animal models ${ }^{15,16}$ and several clinical trials, ${ }^{17-19}$ which demonstrated the safety and clinical improvement with mesenchymal stem cells, olfactory ensheathing cells, neural progenitor cells, umbilical cord blood stem cells, and neural stem cell-like cells. ${ }^{18-21}$

It has become obvious that along with the stem cells, the monocytes/macrophages may be implicated in the repair of the brain tissue and cerebral vasculature. ${ }^{22-24}$ Macrophages have been classified as either M1 or M2 polarized populations. Whereas M1 macrophages are powerful inflammatory cells that produce proinflammatory cytokines and phagocytize pathogens, M2 polarized macrophages modulate the inflammatory responses and stimulate angiogenesis and tissue repair. ${ }^{25,26}$ In the central nervous system (CNS), local microglia and newly recruited circulating blood monocytes can polarize toward the M2 cells and ameliorate CNS damage. The capacity of macrophages to stimulate neural repair was firstly demonstrated in spinal cord injury models. ${ }^{27-29}$ Later, experimental studies in stroke model also demonstrated that M1 polarized macrophages are neurotoxic, whereas M2 cells protect the neurons from apoptosis, ${ }^{30}$ and that administration of monocytes as a part of human umbilical cord blood monocytes reduces the infarct size and restores motor function. ${ }^{24}$ Similarly, the recovery of astrocytes, oligodendrocytes, endothelial cells, and myelin in the lipopolysaccharide-injured brain was shown to occur after infiltration of monocytes. ${ }^{31}$

The clinical data available to date have demonstrated a therapeutic potential of stem cells, ${ }^{32}$ but there are no clinical reports on macrophages in the treatment of CP. Nevertheless, several groups have proposed that cell products composed of human monocytes could be considered as candidates for the treatment of injury-induced CNS demyelination. ${ }^{23,33,34}$

Recently, we designed a new protocol for the generation of M2-like macrophages using low growth factor conditions. ${ }^{35}$ These cells differ from M1 cells by higher expression of M2-accosiated (CD206) and proapoptogenic (B7-H1, TRAIL, FasL) molecules and lower production of many proinflammatory cytokines and chemokines. In addition, these macrophages are unable to stimulate effective T-cell proliferation in mixed lymphocyte reaction as well as to induce Th1/Th17 response, and possess considerable regenerative potential (in particular, produce high amounts of insulin-like growth factor 1 and vascular endothelial growth factor). This study aimed to assess the safety of M2 cell transplantation in severe CP patients and the efficacy of transplanted M2 cells in neurologic improvement.

\section{Patients and methods}

\section{Study design and patient enrollment}

An open-label, Phase I/II, non-controlled clinical study of chronic children who had severe CP was conducted. The purpose of this study was to assess the safety and therapeutic efficacy of M2-like macrophages for treatment of CP patients. A written informed consent was obtained from the patients' parents before the therapy. Permission for this study was obtained from the Academic Board and Review Board (Local Ethics Committee) of the Research Institute of Fundamental and Clinical Immunology (Novosibirsk, Russia).

Patients with CP were considered eligible if they fulfilled the following inclusion criteria: 1 ) age $\geq 12$ months and $\leq 10$ years; 2) performance status: Gross Motor Function Classification System - level IV-V; 3) parental consent. The exclusion criteria were: 1) autism and autistic spectrum disorders without motor disability; 2) progressive neurologic disease; 3) human immunodeficiency virus or uncontrolled bacterial, fungal, or viral infections; 4) impaired renal or liver function; 5) genetic disease or phenotypic evidence of a genetic disease on physical examination; 6) requires ventilatory support; and 7) unable to obtain parental consent.

\section{Macrophage generation and introduction}

The generation of macrophages from peripheral blood plasticadherent cells was performed according to the previously developed protocol. ${ }^{35}$ In brief, adherent cells were cultured in RPMI-1640 (BioloT, St. Petersburg, Russia) with supplements at $37^{\circ} \mathrm{C}$ with $5 \% \mathrm{CO}_{2}$. To obtain M2-like macrophages, we used recombinant human granulocyte-macrophage colony-stimulating factor (50 ng/mL; R\&D Systems, Inc., 
Minneapolis, MN, USA) and serum deprivation conditions (low percentage of autologous plasma). In 7 days, the macrophages were harvested by using EDTA in Hanks' balanced salt solution (all reagents from Sigma-Aldrich Co., St Louis, MO, USA), washed, and counted. Then, the generated M2-like macrophages were resuspended in $2 \mathrm{~mL}$ sodium chloride $0.9 \%$ and infused into spinal cord fluid of the patient.

\section{Measurement of safety and efficacy}

All patients were estimated before cell therapy, 3 months after cell therapy, and followed up for the following 5-year period. The primary outcome measure was safety, which included assessment of mortality of any cause and immediate adverse reactions as well as long-term side effects and comorbidities. The secondary outcome measure was functional improvement in neurologic scales, including Gross Motor Function Measure (66-item GMFM) test, Peabody Developmental Motor Scale-Fine Motor test, Ashworth scale, Medical Research Council (MRC) scale, and easy-to-understand unified questionnaire for evaluation of cognitive functions in our modification. Gross motor abilities were characterized by six functions (head controlling, rolling, crawling, sitting, standing, and walking). Fine motor development was evaluated on five functions (hand movement in the midline, active grasping of objects, shifting the objects from one hand to the other, pinching of tiny objects, and "eye-hand" coordination). Five-point Ashworth scale was used for evaluating the degree of spasticity, and six-point MRC weakness scale served for muscle strength estimation. Cognitive functions were estimated based on six functions (understanding addressed speech, speaking of single words, aggression, contact with outsiders, recognizing relatives, and meaningful glance).

To evaluate the influence of M2 therapy on endogenous production of brain-derived neurotrophic factor (BDNF), the samples of patient serum were collected before and a month after macrophage introduction and frozen at $-80^{\circ} \mathrm{C}$ until the measurement. The concentration of BDNF was determined using enzyme-linked immunosorbent assay following the instructions of manufacturers (R\&D Systems, Inc.).

\section{Statistical analysis}

Data were expressed as means \pm standard error and median (Me) with interquartile range (lower quartile-upper quartile). Statistica 6.0 software for Windows (StatSoft Inc., Tulsa, OK, USA) was used for analysis of data. Fisher's exact test was used to determine the relationships between categorical variables. Wilcoxon matched pairs test and Mann-Whitney $U$ test were used to compare nonparametric values.

\section{Results}

Fifty-seven patients (33 boys and 24 girls) with severe CP were enrolled in the current study (Table 1). The Me age was 4 years (range $1-10$ years). The vast majority of children $(84 \%)$ had the fifth level of movement abnormalities estimated by Gross Motor Function Classification System. The types of CP were as follows: 25 spastic quadriplegia, 26 double hemiplegia, 6 others (ataxic, athetoid, triplegia). According to the computed tomography findings, the leading cause of CP was brain atrophy (65\%), indicative of hypoxic-ischemic brain damage, along with intracranial hemorrhage (9\%) and unknown causes $(26 \%)$.

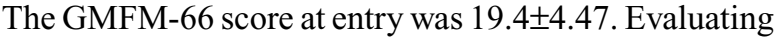
the degree of spasticity based on Ashworth scale evidenced a considerable (4-5 point) increase in muscle tone in 39/57 (68\%) CP children with an average Ashworth score of 3.5 \pm 0.11 . The MRC weakness score reflecting muscle strength in forearms was $1.28 \pm 0.14$, which indicated a marked reduction in muscle strength in all children at baseline. Twenty-nine children had epileptic seizures requiring anticonvulsant therapy. Mental faculties were impaired in practically all patients. Actually, 48 patients (84\%) were unable to speak and 42 children ( $73 \%$ ) could not understand the addressed speech.

All patients received one grafting of autologous M2-like macrophages. On average, $11.6 \pm 0.86 \times 10^{6} \mathrm{M} 2$-like

Table I Baseline characteristics of 57 patients

\begin{tabular}{ll}
\hline Parameters & Mean \pm SEM (Me; LQ-UQ) \\
\hline Age, years & $4.8 \pm 0.33(4 ; 3-7)$ \\
Boys/girls & $33 / 24$ \\
Body weight, kg & $15.6 \pm 1.05(13 ; 12-17)$ \\
CP type & \\
$\quad$ Spastic quadriplegia & $24(42.1 \%)$ \\
$\quad$ Double hemiplegia & $26(45.6 \%)$ \\
$\quad$ Athetoid quadriplegia & $2(3.5 \%)$ \\
$\quad$ Mixed quadriplegia & $5(8.8 \%)$ \\
GMFCS & \\
$\quad$ Level III & $3(5.3 \%)$ \\
$\quad$ Level IV & $6(10.5 \%)$ \\
$\quad$ Level V & $48(84.2 \%)$ \\
Seizures & $29 / 57(50.8 \%)$ \\
GMFM & $19.4 \pm 4.47(0 ; 0-28)$ \\
Ashworth scale & $3.5 \pm 0.11(4 ; 3-4)$ \\
MRC scale & $1.28 \pm 0.14(1 ; 0-2)$ \\
Fine motor, PDMS-FM & $0.9 \pm 0.23(0 ; 0-0)$ \\
Cognition & $1.22 \pm 0.24(0 ; 0-2)$ \\
Cell number, I0 & $11.6 \pm 0.86(11.2 ; 5.2-16.2)$ \\
\hline
\end{tabular}

Abbreviations: CP, cerebral palsy; GMFCS, Gross Motor Function Classification System; LQ-UQ, lower quartile-upper quartile; Me, median; MRC, Medical Research Council; PDMS-FM, Peabody Developmental Motor Scale-Fine Motor; SEM, standard error of the mean. 


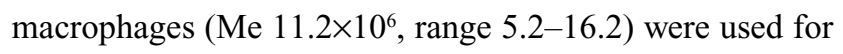
intrathecal introduction. The viability of obtained cells in all cases exceeded 93\%. Patients were evaluated for neurologic changes 3 months after therapy and further monitored up to 5 years. An overall improvement was defined as a score change in GMFM $>4$ points.

Endolumbar administration of M2-like cells was generally well tolerated. After transplantation, mild fever was observed in 31 patients (54\%) and 13 patients $(22.8 \%)$ had intermittent vomiting. These cell therapy-related reactions were only observed during the first 2 days and were well managed with medications Dexasone (KRKA Pharma, Moscow, Russia), Metoclopramide (AWD Pharma GmbH \& Co. KG, Dresden, Germany). No cases of immediate severe adverse events such as allergic reactions, local hematoma or infections at the site of lumbar puncture, meningeal reactions, and systemic inflammatory or toxic complications were noted after cell introduction. At the 3-month follow-up, no signs of neurologic worsening, appearance of new seizure episodes, or increase in the frequency of existing episodes, as well as other serious comorbidities (dyspepsia, infections, and so on) were observed. However, one child demonstrated exacerbation of atopic dermatitis. Till the end of follow-up period, all patients were alive and had no long-term comorbidities and tumors.

After 3 months, all scales showed remarkable improvement from the baseline score. Overall therapeutic responses are shown in Table 2 and Figure 1. It is evident from Table 2 that gross and fine motor activities as well as the muscle strength were enhanced significantly $(p<0.01)$. In addition, a significant decrease in spasticity was also observed at 3 months after transplantation $(p<0.01)$. Finally, cell therapy resulted in obvious improvement of cognitive functions $(p<0.01)$.

Favorable clinical outcome was noted in $42 / 57$ cellgrafted CP children (73\%; responding group). The majority of children enrolled in our investigation could not independently keep their head in a vertical position $(41 / 57 ; 72 \%)$

Table 2 Neurologic examination of CP children $(\mathrm{N}=57)$ over a 3-month observation period after M2 macrophage introduction

\begin{tabular}{lll}
\hline Scales & $\begin{array}{l}\text { Before M2 } \\
\text { introduction }\end{array}$ & $\begin{array}{l}\text { After M2 } \\
\text { introduction }\end{array}$ \\
\hline GMFM-66 & $19.4 \pm 4.47(0 ; 0-28)$ & $73 \pm 7.8(64 ; 8-124)^{* *}$ \\
Ashworth & $3.5 \pm 0.11(4 ; 3-4)$ & $2.5 \pm 0.16(2 ; 1-4)^{* *}$ \\
MRC & $1.28 \pm 0.14(1 ; 0-2)$ & $2.5 \pm 0.15(3 ; 2-3)^{*}$ \\
Fine motor activity & $0.9 \pm 0.23(0 ; 0-0)$ & $4.4 \pm 0.51(3 ; 0-8)^{* *}$ \\
Cognition & $1.22 \pm 0.24(0 ; 0-2)$ & $3.98 \pm 0.95(4 ; 2.5-5.5)^{* *}$ \\
\hline
\end{tabular}

Notes: $* p<0.05, * * p<0.01$ (Wilcoxon matched pairs test).

Abbreviations: CP, cerebral palsy; GMFM, Gross Motor Function Measure; MRC, Medical Research Council. and had no capacity to crawl and roll over (48/57; 84\%). With M2 therapy, 34 children out of $41(82 \%)$ were able to keep the head and 31 children (64\%) were able to crawl. Moreover, CP children treated with M2 cells displayed an obvious improvement in toy manipulations, grasping, and visual-motor integration (Figure 1). The beneficial effects of cell therapy appeared not only in motor activities, but also in cognitive/mental functions. In fact, we observed a decrease in aggression (38/42) and improvement of contact with outsiders (34/52). Sixteen of 42 children understood or improved their understanding of the addressed speech, and 12 of 51 children showed the appearance of a meaning-bearing speech. Of importance, 10/29 children (34\%) with seizure syndrome (up to 10 episodes per month) experienced seizure arrest, and another 14 children exhibited significantly reduced seizure frequency (from 7-10 to 1-2 episodes per month). The improvement of motor functions and mental abilities appeared quite early, from several days to 1 month, was clearly manifested at 3 months, and did not reduce over time.

By comparing the children responding and not responding to cell therapy, we found that the better outcomes did not depend on some demographic parameters (age or sex) and severity of neurologic deficit as well as the number of input cells. However, the children who responded to cell therapy showed a trend to have higher GMFM scores than the nonresponders (23.2 \pm 5.5 vs $6.8 \pm 6.8, p=0.066)$.

Finally, to evaluate the influence of M2 therapy on endogenous neurotrophic factor production, we examined the concentration of BDNF in the serum of CP children 1 month after M2 administration. The data showed that the macrophage injection was accompanied by an increased production of BDNF (from $695 \pm 60$ to $1183 \pm 153 \mathrm{pg} / \mathrm{mL} ; \mathrm{p}_{\mathrm{U}}=0.015$ ), which was more pronounced in the responding group.

\section{Discussion}

Recent studies have demonstrated that cell-based therapy can improve neurologic functions in various neuropathologies including CP. ${ }^{14,36,37}$ Along with stem cells and their derivatives, a major role of M2 anti-inflammatory macrophages in promoting neural repair was documented. ${ }^{27,29}$ The idea on the beneficial role of macrophages in CNS repair was firstly proposed by the group of Michal Schwartz which demonstrated that implantation of the macrophages into transected rat spinal cord stimulated tissue repair and partial recovery of motor function. ${ }^{38}$ Later, this group initiated a pilot Phase I study of M2-like macrophage implantation in patients with acute complete spinal cord injury ${ }^{38}$ and a Phase II randomized controlled multicenter trial. ${ }^{40}$ Despite 


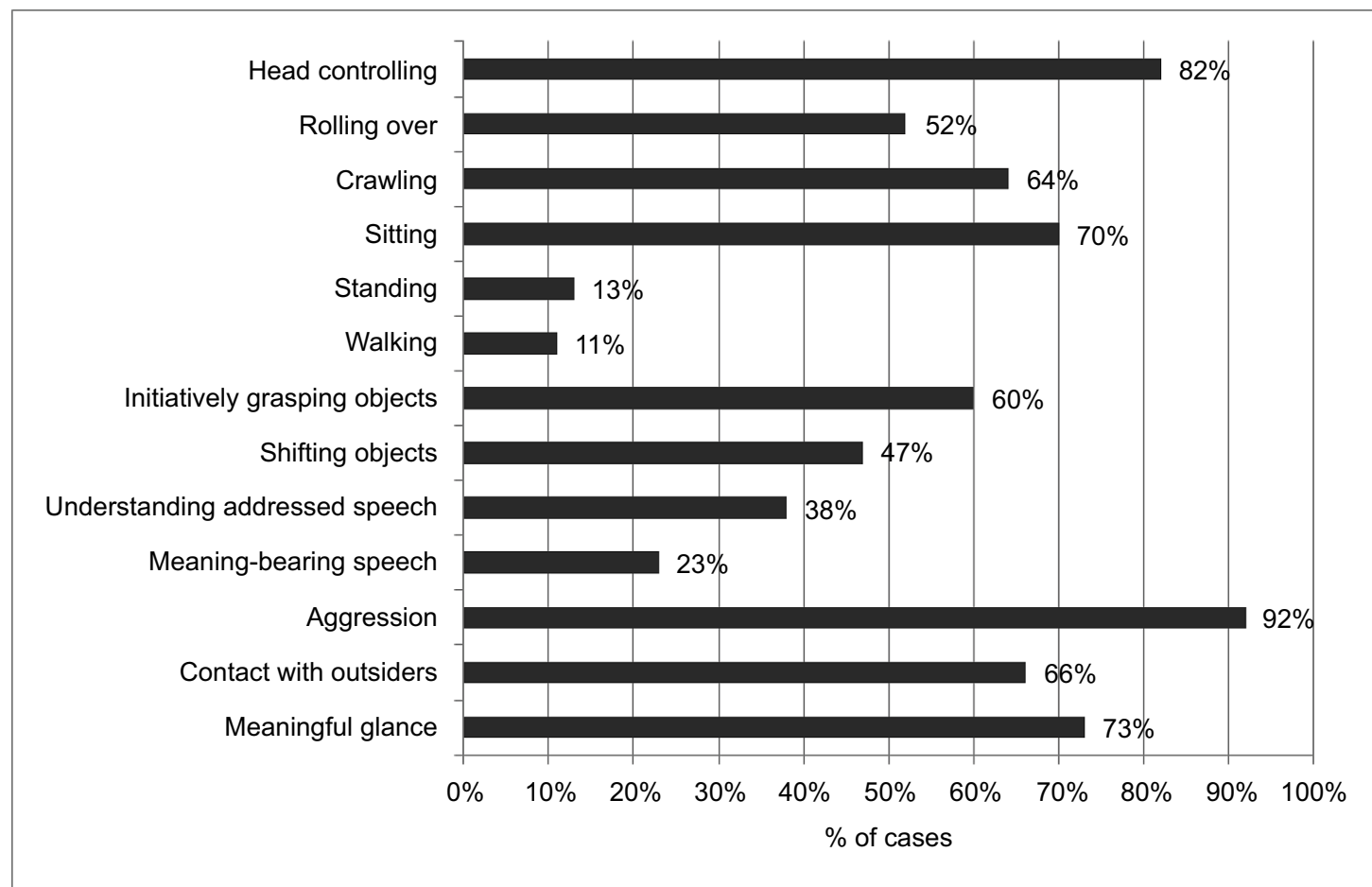

Figure I The improvement in motor and mental activities in CP patients at 3 months after M2 macrophage treatment. Note: Data are presented as the percentage of positive cases in patients who initially were unable to perform the function. Abbreviation: $\mathrm{CP}$, cerebral palsy.

the lack of an obvious clinical improvement due largely to the invasiveness of implanting procedure, the clinical trial was a major achievement to transfer macrophage-based therapy into clinic. Immunomodulation by M2 macrophages may partially underlie the effect of stem cell-based therapy, since many transplanted cells die from apoptosis and engulfment of apoptotic cells by macrophages induces M2 polarization. ${ }^{41}$ Another possibility is that transplanted mesenchymal stem cells can "educate" macrophages toward an immunoregulatory phenotype. ${ }^{42}$

This study provides the first evidence of the possible application of M2 macrophages for the treatment of CP. We utilized our original approach to obtained M2 cells using low growth factor conditions ${ }^{35}$ and have shown that M2 macrophages may be successfully generated in children with severe CP. Intrathecal introduction of these M2 macrophages was shown to be safe, well tolerated, and did not result in immediate adverse reactions and long-term side effects and comorbidities. In one patient, an aggravation of atopic dermatitis was registered, and we attribute this to the possible Th2-stimulating capacity of M2 ${ }^{43}$ Certainly, this fact requires a careful examination of patients for allergic diseases and may be the exclusion criterion for M2 macrophage application in children with severe and diffuse forms of atopic pathology. On the other hand, exacerbation of atopic dermatitis in only
1/57 patients evidenced that endolumbar application of macrophages did not induce systemic activation of Th2 response. The important result of this study is the analysis of long-term adverse effects after 5-year follow-up demonstrating that there was no case of neurologic worsening, appearance of new episodes or enhancement of seizures, or development of tumor during this period.

In our study, there was no control group and the only possibility to evaluate the effect of transplanted cells was the comparison of motor function and cognitive abilities before and after therapy. M2 macrophage implantation was accompanied by significant decrease in spasticity and enhancement of muscle strength and GMFM scores along with a marked improvement in cognitive functions. Importantly, functional improvement appeared quite early (from some days to 1 month), which was consistent with the observations of other researchers. ${ }^{18-20}$ Once the improvements appeared, they did not decrease with time. Given that the majority of patients recruited to our clinical trial had the fifth level of disability, did not display any improvement after 3-6 months of standard rehabilitation training before cell therapy, were older than 3 years ( $64 \%$ of patients), and displayed early manifestation of the improvement, we suggest that the positive effects were not the result of rehabilitation therapy or age-related development, but were primarily mediated by transplanted 
cells. Of importance is the fact that M2 therapy ameliorates seizure syndrome. This is confirmed by full arrest of seizures in 10 persons and pronounced decrease in the rate of seizure episodes in another 14 children out of 29 patients having seizure syndrome.

Our results concerning the possibility of cell therapy to improve psychomotor functions in $\mathrm{CP}$ are consistent with others. Chen et al showed that fetal olfactory ensheathing cells (OECs) injected into the bilateral corona radiata in the frontal lobes resulted in a significant increase in GMFM score and improvement of mental functions in $\mathrm{CP} .{ }^{18}$ Luan et al demonstrated similar results utilizing intracerebral implantation of allogeneic neural progenitor cells. ${ }^{19}$ The efficacy of cord blood stem cells was demonstrated by Min et al, who showed amelioration of motor and cognitive impairments following infusion of allogeneic umbilical cord blood potentiated with recombinant human erythropoietin. ${ }^{21}$

The mechanisms by which M2 macrophages promote neurologic improvement in $\mathrm{CP}$ patients are not quite clear. According to our previous data, M2 cells are capable of spontaneous production of BDNF, insulin-like growth factor 1 , epidermal growth factor (EGF), basic fibroblast growth factor (FGFb), granulocyte-colony stimulating factor (G-CSF), erythropoietin, and vascular endothelial growth factor ${ }^{35}$ that possess neuroprotective activity and stimulate CNS regeneration. ${ }^{44-47}$ Therefore, our findings concerning increase in serum BDNF after cell therapy can argue this hypothesis. Recent findings also showed the capacity of monocytes/ macrophages to differentiate into endothelium-like cells and function as precursors of endothelial cells ${ }^{48}$ participating in vascular repair. ${ }^{49}$ A rapid functional improvement is probably related to the activation of "silent" synapses. Finally, given the major role of inflammatory mediators in the pathogenesis of $\mathrm{CP},{ }^{10}$ a functional improvement may be mediated by the immunomodulatory activity of M2 cells. Reduction of neuroinflammation in the models of CP leads to an improvement in motor function. In this regard, it should be noted that the M2 cells generated in our protocol differ from M1 cells by higher expression of several proapoptogenic molecules (B7-H1, TRAIL, and FasL), which can induce apoptosis of activated immune cells and downregulate the inflammatory response. In any case, further investigations are required to better understand the precise mechanisms underlying the therapeutic effects of $\mathrm{M} 2$ cells.

The advantages of M2 macrophages are the safety and feasibility of the procedure, since it involves utilization of autologous cells generated from peripheral blood, and intrathecal delivery that is less invasive as compared to intra- cerebral implantation of candidate cells. Another privilege is that M2 transplantation has a stable effect and may be carried out in children with seizure syndrome. However, to better define the therapeutic effects of M2 macrophages and to elucidate the mechanism of M2 cells in CP, further studies should be performed.

\section{Conclusion}

The data obtained suggest that cell therapy based on M2 macrophage administration is safe, does not induce any severe cell-related reactions or long-term side effects and comorbidities, and is accompanied by significant neurologic improvements in severe CP patients.

\section{Disclosure}

The authors report no conflicts of interest in this work.

\section{References}

1. Pabon MM, Borlongan CV. Advances in the cell-based treatment of neonatal hypoxic-ischemic brain injury. Future Neurol. 2013;8(2):193-203.

2. Aisen ML, Kerkovich D, Mast J, et al. Cerebral palsy: clinical care and neurological rehabilitation. Lancet Neurol. 2011;10(9): 844-852.

3. Pellegrino L. Cerebral palsy. In: Batshaw ML, editor. When your child has a disability: the complete sourcebook of daily and medical care. Baltimore, MD: Paul H. Brookes Publishing Co.; 2000:275-287.

4. Westbom L, Hagglund G, Nordmark E. Cerebral palsy in a total population of 4-11 year olds in Southern Sweden. Prevalence and distribution according to different $\mathrm{CP}$ classification systems. $B M C$ Pediatr. 2007;7:41

5. Di H, He Q, Liao Y, Kalionis B, Tai X. The role of inflammatory cytokines in the pathogenesis of cerebral palsy. Gynecol Obstet (Sunnyvale). 2016;6:360.

6. Leviton A, Allred EN, Dammann O, et al; ELGAN Study Investigators. Systemic inflammation, intraventricular hemorrhage, and white matter injury. J Child Neurol. 2013;28(12):1637-1645

7. Patra A, Huang H, Bauer JA, Giannone PJ. Neurological consequences of systemic inflammation in the premature neonate. Neural Regen Res. 2017;12(6):890-896.

8. Gabriel ML, Braga FB, Cardoso MR, Lopes AC, Piatto VB, Souza AS. The association between pro- and anti-inflammatory cytokine polymorphisms and periventricular leukomalacia in newborns with hypoxic-ischemic encephalopathy. J Inflamm Res. 2016;9: 59-67.

9. O'Shea TM. Diagnosis, treatment, and prevention of cerebral palsy. Clin Obstet Gynecol. 2008;51(4):816-828.

10. McAdams RM, Juul SE. The role of cytokines and inflammatory cells in perinatal brain injury. Neurol Res Int. 2012;2012:561494.

11. Kannan S, Dai H, Navath RS, et al. Dendrimer-based postnatal therapy for neuroinflammation and cerebral palsy in a rabbit model. Sci Transl Med. 2012;4(130):130ra46

12. Holt RL, Mikati MA. Care for child development: basic science rationale and effects of interventions. Pediatr Neurol. 2011;44(4):239-253.

13. Bartley J, Carroll JE. Stem cell therapy for cerebral palsy. Expert Opin Biol Ther. 2003;3(4):541-549.

14. Carroll JE, Mays RW. Update on stem cell therapy for cerebral palsy. Expert Opin Biol Ther. 2011;11(4):463-471.

15. Meier C, Middelanis J, Wasielewski B, et al. Spastic paresis after perinatal brain damage in rats is reduced by human cord blood mononuclear cells. Pediatr Res. 2006;59(2):244-249. 
16. Leong WK, Lewis MD, Koblar SA. Concise review: preclinical studies on human cell-based therapy in rodent ischemic stroke models: where are we now after a decade? Stem Cells. 2013;31(6):1040-1043.

17. Carroll JE, Borlongan CV. Adult stem cell therapy for acute brain injury in children. CNS Neurol Disord Drug Targets. 2008;7(4):361-369.

18. Chen L, Huang H, Xi H, et al. Intracranial transplant of olfactory ensheathing cells in children and adolescents with cerebral palsy: a randomized controlled clinical trial. Cell Transplant. 2010;19(2):185-191.

19. Luan Z, Liu W, Qu S, et al. Effects of neural progenitor cell transplantation in children with severe cerebral palsy. Cell Transplant. 2012;Suppl 1:S91-S98.

20. Chen G, Wang Y, Xu Z, et al. Neural stem cell-like cells derived from autologous bone mesenchymal stem cells for the treatment of patients with cerebral palsy. J Transl Med. 2013;11:21

21. Min K, Song J, Kang JY, et al. Umbilical cord blood therapy potentiated with erythropoietin for children with cerebral palsy: a double-blind, randomized, placebo-controlled trial. Stem Cells. 2013;31(3):581-591.

22. Glod J, Kobiler D, Noel M, et al. Monocytes form a vascular barrier and participate in vessel repair after brain injury. Blood. 2006;107(3):940-946.

23. Sanberg PR, Park DH, Kuzmin-Nichols N, et al. Monocyte transplantation for neural and cardiovascular ischemia repair. J Cell Mol Med. 2010;14(3):553-563

24. Womble TA, Green S, Shahaduzzaman M. Monocytes are essential for the neuroprotective effect of human cord blood cells following middle cerebral artery occlusion in rat. Mol Cell Neurosci. 2014;59:76-84.

25. Gordon S, Taylor PR. Monocyte and macrophage heterogeneity. Nat Rev Immunol. 2005;5(12):953-964.

26. Sica A, Mantovani A. Macrophage plasticity and polarization: in vivo veritas. J Clin Invest. 2012;122(3):787-795.

27. Kigerl KA, Gensel JC, Ankeny DP, Alexander JK, Donnelly DJ, Popovich PG. Identification of two distinct macrophage subsets with divergent effects causing either neurotoxicity or regeneration in the injured mouse spinal cord. J Neurosci. 2009;29(43):13435-13444.

28. Prewitt CM, Niesman IR, Kane CJ, Houle JD. Activated macrophage/ microglial cells can promote the regeneration of sensory axons into the injured spinal cord. Exp Neurol. 1997;148(2):433-443.

29. Shechter R, London A, Varol C, et al. Infiltrating blood-derived macrophages are vital cells playing an anti-inflammatory role in recovery from spinal cord injury in mice. PLoS Med. 2009;6(7):e1000113.

30. Hu X, Li P, Guo Y, et al. Microglia/macrophage polarization dynamics reveal novel mechanism of injury expansion after focal cerebral ischemia. Stroke. 2012;43(11):3063-3070.

31. Jeong HK, Ji KM, Kim J, Jou I, Joe EH. Repair of astrocytes, blood vessels, and myelin in the injured brain: possible roles of blood monocytes. Mol Brain. 2013;6:28.

32. Jeong H, Yim HW, Cho YS et al. Efficacy and safety of stem cell therapies for patients with stroke: a systematic review and single arm meta-analysis. Int J Stem Cells. 2014;7(2):63-69.
33. Shechter R, Schwartz M. Harnessing monocyte-derived macrophages to control central nervous system pathologies: no longer 'if' but 'how'. J Pathol. 2013;229(2):332-346.

34. Kurtzberg J, Buntz S, Gentry T, et al. Preclinical characterization of DUOC01 , a cell therapy product derived from banked umbilical cord blood for use as an adjuvant to umbilical cord blood transplantation for treatment of inherited metabolic diseases. Cytotherapy. 2015;17(6):803-815.

35. Chernykh ER, Shevela EY, Sakhno LV, Tikhonova MA, Petrovsky YL, Ostanin AA. The generation and properties of human M2-like macrophages: potential candidates for CNS repair? Cell Ther Transplant. 2010;2:e.000080.01

36. Aboody K, Capela A, Niazi N, Stern JH. Temple S. Translating stem cell studies to the clinic for CNS repair: current state of the art and the need for a Rosetta stone. Neuron. 2011;70(4):597-613.

37. Tate CC, Case CC. Mesenchymal stromal cells to treat brain injury. Advanced Topics in Neurological Disorders, ISBN: 978-953-51-0303-5.

38. Rapalino O, Lazarov-Spiegler O, Agranov E, et al. Implantation of stimulated homologous macrophages results in partial recovery of paraplegic rats. Nat Med. 1998;4(7):814-821.

39. Knoller N, Auerbach G, Fulga V, et al. Clinical experience using incubated autologous macrophages as a treatment for complete spinal cord injury: phase I study results. J Neurosurg Spine. 2005;3(3):173-181.

40. Lammertse DP, Jones LA, Charlifue SB, et al. Autologous incubated macrophage therapy in acute, complete spinal cord injury: results of the phase 2 randomized controlled multicenter trial. Spinal Cord. 2012;50(9):661-671.

41. Thum T, Bauersachs J, Poole-Wilson PA, Volk HD, Anker SD. The dying stem cell hypothesis: immune modulation as a novel mechanism for progenitor cell therapy in cardiac muscle. J Am Coll Cardiol. 2005;46(10):1799-1802.

42. Eggenhofer E, Hoogduijn MJ. Mesenchymal stem cell-educated macrophages. Transplant Res. 2012;1:12.

43. Mills CD. M1 and M2 macrophages: oracles of health and disease. Crit Rev Immunol. 2012;32(6):463-488.

44. Varon SS, Conner JM, Kuang RZ. Neurotrophic factors: repair and regeneration in the central nervous system. Restor Neurol Neurosci. 1995;8(1):85-94.

45. Carro E, Trejo JL, Núñez A, Torres-Aleman I. Brain repair and neuroprotection by serum insulin-like growth factor-I. Mol Neurobiol. 2003;27(2):153-162.

46. King CE, Rodger J, Bartlett C, Esmaili T, Dunlop SA, Beazley LD. Erythropoietin is both neuroprotective and neuroregenerative following optic nerve transection. Exp Neurol. 2007;205(1):48-55.

47. Storkebaum E, Carmeliet P. VEGF: a critical player in neurodegeneration. J Clin Invest. 2004;113(1):14-18.

48. Rehman J, Li J, Orschell CM, March KL. Peripheral blood "endothelial progenitor cells" are derived from monocyte/macrophages and secrete angiogenic growth factors. Circulation. 2003;107(8):1164-1169.

49. Glod J, Kobiler D, Noel M, et al. Monocytes form a vascular barrier and participate in vessel repair after brain injury. Blood. 2006;107(3):940-946.
Journal of Neurorestoratology

\section{Publish your work in this journal}

The Journal of Neurorestoratology is an international, peer-reviewed open access online journal publishing original research and review articles on the subject of Neurorestoratology. To provide complete coverage of this revolutionary field the Journal of Neurorestoratology will report on relevant experimental research, technological advances, and clinical achievements. The manuscript management system is completely online and includes a very quick and fair peer-review system, which is all easy to use. Visit http://www.dovepress.com/testimonials. php to read real quotes from published authors. 